TRANSACTIONS OF THE

AMERICAN MATHEMATICAL SOCIETY

Volume 352, Number 11, Pages 5263-5277

S 0002-9947(00)02620-9

Article electronically published on July 18, 2000

\title{
A CLASSIFICATION OF ONE DIMENSIONAL ALMOST PERIODIC TILINGS ARISING FROM THE PROJECTION METHOD
}

\author{
JAMES A. MINGO
}

\begin{abstract}
For each irrational number $\alpha$, with continued fraction expansion $\left[0 ; a_{1}, a_{2}, a_{3}, \ldots\right]$, we classify, up to translation, the one dimensional almost periodic tilings which can be constructed by the projection method starting with a line of slope $\alpha$. The invariant is a sequence of integers in the space $X_{\alpha}=\left\{\left(x_{i}\right)_{i=1}^{\infty} \mid x_{i} \in\left\{0,1,2, \ldots, a_{i}\right\}\right.$ and $x_{i+1}=0$ whenever $\left.x_{i}=a_{i}\right\}$ modulo the equivalence relation generated by tail equivalence and $\left(a_{1}, 0, a_{3}, 0, \ldots\right) \sim$ $\left(0, a_{2}, 0, a_{4}, \ldots\right) \sim\left(a_{1}-1, a_{2}-1, a_{3}-1, \ldots\right)$. Each tile in a tiling $\mathrm{T}$, of slope $\alpha$, is coded by an integer $0 \leq x \leq[\alpha]$. Using a composition operation, we produce a sequence of tilings $\mathrm{T}_{1}=\mathrm{T}, \mathrm{T}_{2}, \mathrm{~T}_{3}, \ldots$. Each tile in $\mathrm{T}_{i}$ gets absorbed into a tile in $\mathrm{T}_{i+1}$. A choice of a starting tile in $\mathrm{T}_{1}$ will thus produce a sequence in $X_{\alpha}$. This is the invariant.
\end{abstract}

The subject of this paper is the classification one dimensional almost periodic tilings obtained by the projection method. These sequences were extensively studied by Morse and Hedlund under the name of Sturmian trajectories; but there are earlier works by J. Bernoulli [JB], H. J. S. Smith [HJSS], and E. Christoffel [EBC] among others. For a survey of the recent literature see the references in the papers of T. Brown [TCB] and Lunnon and Pleasants [LP].

There are a number of axiomatic characterizations of these sequences. We have found it convenient to use one found by C. Series [CS] (which is essentially that of Morse and Hedlund). The equivalence of this with other characterizations is presented in [LP]. We shall review below the axioms of Series.

With each tiling there is an associated real number $\alpha$, which we shall call the slope of the tiling; the continued fraction expansion of $\alpha$ is reflected in the structure of the tiling [CS]. The tilings can be grouped into families, $\mathcal{T}_{\alpha}$, in which all members have the same slope. Moreover there is a simple geometric construction for obtaining members of $\mathcal{T}_{\alpha}$ using the projection method with lines of slope $\alpha$.

We shall be concerned with the case when $0<\alpha<1$ and $\alpha$ is irrational. In this case the members of $\mathcal{T}_{\alpha}$ have a property characteristic of almost periodic tilings: if $\mathrm{T}_{1}$ and $\mathrm{T}_{2}$ are in the family $\mathcal{T}_{\alpha}$, then every segment of $\mathrm{T}_{1}$ appears in $\mathrm{T}_{2}$ and appears infinitely often. Thus on a local level all elements of $\mathcal{T}_{\alpha}$ appear similar.

To distinguish the elements of $\mathcal{T}_{\alpha}$ we use a space $X_{\alpha}$ constructed from the continued fraction expansion of $\alpha=\left[0 ; a_{1}, a_{2}, a_{3}, \ldots\right]$. Let $X_{\alpha}=\left\{\left(x_{i}\right)_{i=1}^{\infty} \mid x_{i} \in\right.$ $\left\{0,1, \ldots, a_{i}\right\}$ and $x_{i+1}=0$ whenever $\left.x_{i}=a_{i}\right\}$. From a tiling $\mathrm{T} \in \mathcal{T}_{\alpha}$ and a tile $\mathrm{t} \in \mathrm{T}$

Received by the editors August 4, 1998 and, in revised form, May 1, 1999.

1991 Mathematics Subject Classification. Primary 05B45, 52C22, 46L89.

Research supported by the Natural Sciences and Engineering Research Council of Canada and The Fields Institute for Research in the Mathematical Sciences. 
we construct $x \in X_{\alpha}$, and we show that these classify the tilings up to translation. For certain irrationals this expansion is related to the $\beta$-expansions of Rényi and Parry ( $\mathrm{AR}],[\mathrm{WP}])$.

When $\alpha=(\sqrt{5}-1) / 2$ we have $X_{\alpha}=\left\{\left(x_{i}\right) \mid x_{i} \in\{0,1\}\right.$ and $\left.x_{i}=1 \Rightarrow x_{i+1}=0\right\}$, and $X_{\alpha}$, modulo tail equivalence, was called by Connes [AC] (section II.3) the Penrose universe because it classifies Penrose tilings (see [GS], section 10.6; also [AP] and [EAR], where the dynamical properties of these and other tilings are explored). In $\left[\mathrm{NDB}_{1}\right]$ and $\overline{\mathrm{NDB}_{2}}$ a classification of Beatty sequences (sequences of the form $\left.\{[n \alpha+\beta]\}_{n=1}^{\infty}\right)$ for $\alpha=(\sqrt{5}-1) / 2$ or $\alpha=\sqrt{2}-1$ was discussed. However our method is simpler, more general, and works for every irrational.

\section{INTRODUCTION}

1.1. Definition. A two sided infinite sequence $\left\{\mathrm{t}_{i}\right\}_{i=1}^{\infty}$ is composable if it satisfies the following axioms:

$A_{1}$ : The letter $a$ is isolated: i.e. if $t_{i}=a$ then $t_{i \pm 1}=b$.

$\mathrm{A}_{2}$ : There is an integer $n$ such that between a's there are either $n$ or $n+1$ b's.

If $\mathrm{T}$ is a composable sequence, we can produce a new sequence $\mathrm{T}^{\prime}$ by composition: each segment beginning with an a and followed by $n$ b's gets replaced by a b, and any remaining b's are replaced by an a. Thus

$$
\mathrm{a} \underbrace{\mathrm{bbb} \ldots \mathrm{b}}_{n} \mapsto \mathrm{b} \quad \text { and } \quad \mathrm{a} \underbrace{\mathrm{bbb} \ldots \mathrm{b}}_{n+1} \mapsto \mathrm{ba}
$$

The third axiom asserts that the sequence can be composed infinitely many times.

$\mathrm{A}_{3}$ : Each composition satisfies $\mathrm{A}_{1}$ and $\mathrm{A}_{2}$.

If $\mathrm{T}=\left\{\mathrm{t}_{i}\right\}_{i=1}^{\infty}$ satisfies axioms $\mathrm{A}_{1}, \mathrm{~A}_{2}$, and $\mathrm{A}_{3}$, then we call $\mathrm{T}$ a cutting sequence (following Series $[\overline{\mathrm{CS}}]$ ).

1.2. Remark. As explained in Series [CS], cutting sequences can be constructed as follows. Let $\alpha$ be an irrational number between 0 and 1 and let $\beta$ be any real number. Let $\mathrm{L}$ be the line with equation $y=\alpha x+\beta$. Each intersection of $\mathrm{L}$ with a horizontal line will be marked with an a, and each intersection of $L$ with a vertical line will be marked with $a b$. Thus along $L$ we obtain a two sided infinite sequence $T$ of a's and b's. These are the one dimensional almost periodic sequences obtained by the projection method (see [MS], section 4.3). In [LP], Lemmas 2.2 and 3.3, it is shown that $\mathrm{T}$ is a cutting sequence (see also $[\mathrm{GS}], 10.6 .2$ ).

When a cutting sequence for the line $y=\alpha x+\beta$ is constructed by the projection method, a tiling of the line is produced with a-tiles intervals of length $\sin \vartheta$ and b-tiles intervals of length $\cos \vartheta$, where $\alpha=\tan \vartheta$.

It will be convenient later on to have a notation for labelling the symbols (tiles). The intersection of $\mathrm{L}$ with the horizontal line $y=m$ produces an a tile; let us label it $\mathrm{a}_{m}$. The intersection of $\mathrm{L}$ with the vertical line $x=n$ produces a $\mathrm{b}$ tile; let us label it $\mathrm{b}_{n}$. The intersection of $\mathrm{L}$ with the $y$-axis produces $\mathrm{b}_{0}$. By following the path of $b_{0}$ through successive compositions we shall produce our invariant.

If $L$ passes through a point of $\mathbb{Z}^{2}$ then an a and $a b$ will coincide. We will in this case obtain two sequences $\mathrm{T}^{+}$and $\mathrm{T}^{-} . \mathrm{T}^{+}$is constructed by writing all coinciding pairs $\{a, b\}$ with the a's preceding the b's (i.e. to the left), and $\mathrm{T}^{-}$with the a's following the b's. 
1.3. Definition. Given a cutting sequence $T$, let $T_{1}, T_{2}, \ldots$ be the sequence obtained from $T$ by composition: i.e. $T_{2}$ is obtained from $T_{1}$ by composition, and in general $\mathrm{T}_{i+1}$ is obtained from $\mathrm{T}_{i}$ by composition. Let $a_{i}$ be the integer for $\mathrm{T}_{i}$ postulated by axiom $\mathrm{A}_{2}$. Then $\alpha$, the real number with continued fraction expansion $\left[0 ;, a_{1}, a_{2}, a_{3}, \ldots\right]$, is the slope of $\mathrm{T}$.

1.4. Definition. Let $\mathrm{T}$ be a cutting sequence with slope $\alpha=\left[0 ; a_{1}, a_{2}, a_{3}, \ldots\right]$ and let $\left\{\mathrm{T}_{i}\right\}_{i=1}^{\infty}$ be the sequence of cutting sequences obtained from $\mathrm{T}$ by composition (setting $T_{1}=T$ ). Let $t$ be a letter in $T$ and let $t_{2}$ be the letter in $T_{2}$ into which $t$ is absorbed by composition. In general let $t_{i+1}$ be the letter of $T_{i+1}$ into which $\mathrm{t}_{i}$ gets absorbed by composition. Letting $\mathrm{t}_{1}=\mathrm{t}$ we obtain a sequence $\left\{\mathrm{t}_{i}\right\}_{i=1}^{\infty}$ with $\mathrm{t}_{i} \in \mathrm{T}_{i}$. We construct $x \in X_{\alpha}$ as follows. If $\mathrm{t}_{i}=\mathrm{a}$ then $x_{i}=0$; if $\mathrm{t}_{i}=\mathrm{b}$ then $x_{i}$ is the number of b's between $\mathrm{t}$ and the first a to the left. Then $x=\left(x_{i}\right) \in X_{\alpha}$ is the coding sequence of the pair $(\mathrm{T}, \mathrm{t})$ :

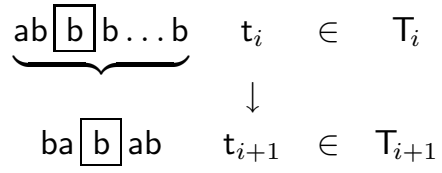

In the example above, $x_{i}=1$ and $x_{i+1}=0$.

We next wish to describe the process by which, given $x \in X_{\alpha}$, one can construct $\mathrm{T} \in \mathcal{T}_{\alpha}$ and $\mathrm{t} \in \mathrm{T}$ such that the coding sequence of $(\mathrm{T}, \mathrm{t})$ is $x$. $\mathrm{T}$ will be written as a limit of words $\left\{T_{1}, T_{2}, T_{3}, \ldots\right\}$ in $\{\mathrm{a}, \mathrm{b}\}$, where each $\mathrm{T}_{i-1}$ is a subword of $\mathrm{T}_{i}$. A special case of this (when $\left.x=\left(a_{1}-1, a_{2}-1, a_{3}-1, \ldots\right)\right)$ was given by Smith [HJSS, and so we shall call $\left\{T_{i}\right\}_{i=0}^{\infty}$ the Smith sequence of $x \in X_{\alpha}$.

Suppose $\alpha=\left[0 ; a_{1}, a_{1}, a_{3}, \ldots\right]$ and $\left(x_{1}, x_{2}, x_{3}, \ldots\right) \in X_{\alpha}$. We shall construct sequences $\left\{S_{-1}, S_{0}, S_{1}, \ldots\right\}$ and $\left\{T_{0}, T_{1}, T_{2}, \ldots\right\}$ of words in the letters $\{\mathrm{a}, \mathrm{b}\}$. We can think of these words as elements in the free group with generators $\{a, b\}$. Let

$$
\begin{aligned}
S_{-1} & =\mathrm{a} \\
S_{0} & =\mathrm{b} \\
S_{1} & =S_{-1} \underbrace{S_{0} \ldots S_{0}}_{a_{1}}=\mathrm{a} \underbrace{\mathrm{b} \ldots \mathrm{b}}_{a_{1}} \\
& \vdots \\
S_{i+1} & =S_{i-1} \underbrace{S_{i} \ldots S_{i}}_{a_{i+1}}
\end{aligned}
$$

Define $T_{0} \subseteq T_{1} \subseteq T_{2} \subseteq \cdots$ by

$$
\begin{aligned}
& T_{0}= \mathrm{b} \\
& T_{1}= \begin{cases}S_{-1} \underbrace{S_{0} \cdots S_{0}}_{x_{1}} T_{0} \underbrace{S_{0} \cdots S_{0}}_{a_{1}-x_{1}-1}=\mathrm{a} \underbrace{\mathrm{b} \cdots \mathrm{b}}_{x_{1}} \underbrace{\mathrm{b} \cdots \mathrm{b}}_{a_{1}-x_{1}-1} & \text { if } x_{1} \neq a_{1} \\
\mathrm{~b} & \text { if } x_{1}=a_{1}\end{cases} \\
& T_{i+1}= \begin{cases}T_{i} & \text { if } x_{i+1}=a_{i+1} \\
S_{i-1} \underbrace{S_{i} \cdots S_{i}}_{x_{i+1}} \underbrace{\underbrace{S_{i} \cdots S_{i}}_{a_{i+1}-x_{i+1}-1}}_{T_{i}} & \text { if } x_{i+1}<a_{i+1} \text { and } x_{i} \neq a_{i} \\
T_{i-1} \underbrace{S_{i} \cdots S_{i}}_{a_{i+1}} & \text { if } x_{i}=a_{i}\end{cases}
\end{aligned}
$$


Note that when $x_{i}=a_{i+i}$ we have $T_{i}=T_{i-1}$, so $T_{i+1}=T_{i} \overbrace{S_{i} \cdots S_{i}}^{a_{i+1}}$; and so in all cases $T_{i}$ is a subword of $T_{i+1}$, the inclusions being indicated by the boxes.

1.5. Definition. We shall call $\left\{T_{i}\right\}$ the Smith sequence of $x \in X_{\alpha}$.

By taking the union $\mathrm{T}$ of the $T_{i}$ 's and $\mathrm{t}=T_{0}$ we have a sequence $\mathrm{T}$ and a tile $\mathrm{t}$ in $\mathrm{T}$. We shall show that $\mathrm{T}$ is a cutting sequence and that the coding sequence of $(\mathrm{T}, \mathrm{t})$ is $x$ (Theorem $\mathrm{C}$ ).

1.6. Examples. Two sequences $x$ and $y$ in $X_{\alpha}$ are if there is an $n$ such that $x_{k}=y_{k}$ for $k \geq n$. If $x \in X_{\alpha}$ is tail equivalent to one of $\left\{-\alpha, 0^{+}, 0^{-}\right\}$, then the limit sequence $T_{0} \rightarrow T_{1} \rightarrow T_{2} \rightarrow \cdots \rightarrow \mathrm{T}$ will not be two sided. In fact, when $x=-\alpha=\left(a_{1}-1, a_{2}-1, a_{3}-1, \ldots\right)$ the sequence produced is the limit of the $T_{i}$ 's:

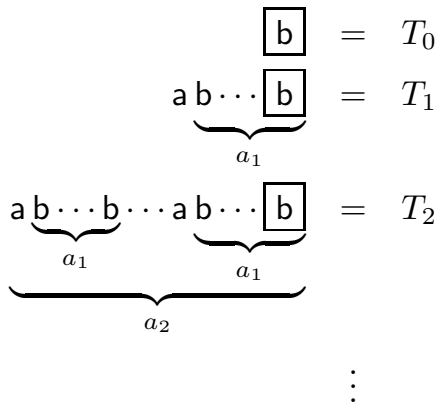

Let us call this sequence $T_{-\alpha}$. This is an infinite sequence extending off to the left, so it it not a cutting sequence, although one can compose it an infinite number of times. If $x=0^{+}=\left(0, a_{2}, 0, a_{4}, \ldots\right)$, then the sequence produced is the limit of the $T_{i}$ 's:

$$
\begin{aligned}
& T_{0}=\mathrm{b} \\
& T_{1}=T_{2}=\mathrm{a} \underbrace{\mathrm{b} b \cdots \mathrm{b}}_{a_{1}} \\
& T_{3}=T_{4}=T_{1} \underbrace{S_{2} \ldots S_{2}}_{a_{3}}
\end{aligned}
$$

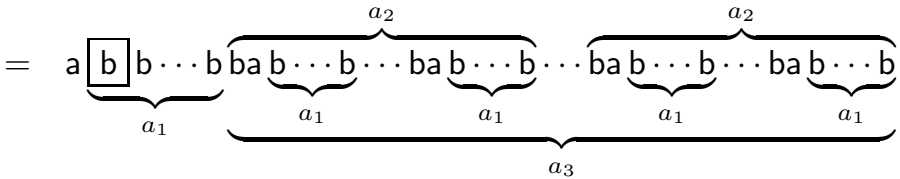

Let us denote this sequence $\mathrm{T}_{0^{+}}$. This infinite sequence, which is also not a cutting sequence, extends off to the right. Finally, suppose $x=0^{-}=\left(a_{1}, 0, a_{3}, 0\right.$, 
...). Then the sequence obtained is

$$
\begin{aligned}
T_{0}=T_{1} & =\mathrm{b} \\
T_{2}=T_{3} & =T_{0} \underbrace{S_{1} \cdots S_{1}}_{a_{2}} \\
& =\mathrm{b} \overbrace{\underbrace{\mathrm{b} \cdots \mathrm{b}}_{a_{1}} \cdots \mathrm{a} \underbrace{\mathrm{b} \cdots \mathrm{b}}_{a_{1}}}^{a_{2}}
\end{aligned}
$$

Let us denote this sequence $\mathrm{T}_{0^{-}}$. This infinite sequence, which is also not a cutting sequence, extends off to the right.

We can assemble $\mathrm{T}_{-\alpha}, \mathrm{T}_{0^{+}}$, and $\mathrm{T}_{0^{-}}$into cutting sequences; however, $\mathrm{T}_{-\alpha} \mid \mathrm{T}_{0^{+}}$ and $T_{-\alpha} \mid T_{0^{-}}$are both cutting sequences (here $\cdot \mid \cdot$ denotes concatenation). The first produces the cutting sequence $\mathrm{T}^{+}$for $\beta=0^{+}$and the second produces $\mathrm{T}^{-}$ for $\beta=0^{-}$. The only difference between $\mathrm{T}_{0^{+}}$and $\mathrm{T}_{0^{-}}$is in the first two letters: the former begins with $a b$ and the latter with $b a$. If we let $T_{-\alpha}^{o p}$ denote the reflection of $T_{-\alpha}$ so as to produce an infinite sequence extending to the right, then $\mathrm{T}_{0^{+}}=\mathrm{ab} \mathrm{T}_{-\alpha}^{\mathrm{op}}$ and $\mathrm{T}_{0^{-}}=\mathrm{baT}_{-\alpha}^{\mathrm{op}}$, and thus $\mathrm{T}^{+}=\mathrm{T}_{-\alpha}|\mathrm{ab}| \mathrm{T}_{-\alpha}^{\mathrm{op}}$ and $\mathrm{T}^{-}=\mathrm{T}_{-\alpha}|\mathrm{ba}| \mathrm{T}_{-\alpha}^{\mathrm{op}}$. We shall call such a pair of cutting sequences conjugate (cf. [LP], section 8). For example, when $\alpha=[0 ; 3,1,3,1, \ldots]$ we have

$$
\begin{aligned}
\mathrm{T}^{+} & =\ldots \text { abbbbabbbbabbb } \mathrm{ab} \text { bbbabbbbabbbabbbb ... } \\
\mathrm{T}^{-} & =\ldots \text { abbbbabbbbabbb } \mathrm{ba} \text { bbbabbbbabbbabbbb ... }
\end{aligned}
$$

1.7. Definition. Let $T$ be a cutting sequence and $t$ a tile in $T$. Let $T_{1}=T$, $T_{2}$ the cutting sequence obtained from $T_{1}$ by composition, and let $t_{2}$ be the tile of $T_{2}$ which absorbs $t_{1}=t$. Iterating this construction, we obtain a sequence $\left\{\left(T_{1}, t_{1}\right),\left(T_{2}, t_{2}\right), \ldots\right\}$ of cutting sequences with a specified letter $t_{i}$ in the cutting sequence $\mathrm{T}_{i}$. From this we may obtain an increasing sequence of words $W_{1} \subseteq W_{2} \subseteq$ $W_{3} \subseteq \cdots \subseteq \mathrm{T}$ as follows. We let $W_{0}=\mathrm{t}_{1}$, and we let $W_{1}$ be all the letters in $\mathrm{T}$ which under composition get absorbed into $t_{2}$. Let $W_{2}$ be all the letters in $T$ which get absorbed, under two compositions, into $t_{3}$. Similarly let $W_{k}$ be all the letters of $\mathbf{T}$ which get absorbed, under $k$ compositions, into $\mathrm{t}_{k+1}$. The sequence $\left\{W_{i}\right\}_{i=1}^{\infty}$ is called the inflation sequence of $(\mathrm{T}, \mathrm{t})$.

Now either $\bigcup_{1=1}^{\infty} W_{i}=\mathrm{T}$, and we shall say that $\mathrm{T}$ is non-singular, or $\bigcup_{i=1}^{\infty} W_{i} \neq$ $\mathrm{T}$, in which case we shall say that $\mathrm{T}$ is singular. By construction $\bigcup_{1=1}^{\infty} W_{i}$ is infinite and contains no gaps, so when $\mathrm{T}$ is singular we can write it as the union of $\bigcup_{1=1}^{\infty} W_{i}$ and $\bigcup_{1=1}^{\infty} W_{i}^{\prime}$, where the $W_{s}^{\prime}$ 's are constructed by choosing another starting tile not in $\bigcup_{1=1}^{\infty} W_{i}$. We shall show in Theorem $\mathrm{C}$ that the Smith sequence reconstructs the inflation sequence. From this it follows that when $\mathrm{T}$ is singular $\bigcup_{i=1}^{\infty} W_{i}$ is either $\mathrm{T}_{-\alpha}, \mathrm{T}_{0^{+}}$, or $\mathrm{T}_{0^{-}}$

The last construction we need tells us which coding sequence is obtained from the line $\mathrm{L}: y=\alpha x+\beta$, when we choose as our initial tile $\mathrm{b}_{0}$ (the intersection of $\mathrm{L}$ with the $y$-axis). To take account of the singular tiles we need to introduce some notation. 
1.8. Notation. Let $\mathbb{R}_{\alpha}$ be the Cantor set obtained by cutting the real line $\mathbb{R}$ at each of the points $\mathbb{Z}+\alpha \mathbb{N}=\{m+\alpha n \mid m, n \in \mathbb{Z}$, and $n \geq 0\}$. Each of the points $m+\alpha n$ of $\mathbb{Z}+\alpha \mathbb{N}$ will be split into two points $(m+\alpha n)^{+}$and $(m+\alpha n)^{-}$.

We can make $\mathbb{R}_{\alpha}$ a locally compact Hausdorff space by writing it as an inverse limit. Let $\left\{\gamma_{n}\right\}_{n=1}^{\infty}$ be an enumeration of $\mathbb{Z}+\alpha \mathbb{N}$. Then $\mathbb{R}_{\alpha}=\lim _{\leftarrow} R_{n}$, where $R_{n}$ is obtained from $R_{n-1}$ by splitting at the point $\gamma_{n}$. The map $R_{n+1} \rightarrow R_{n}$ forgets about the splitting at $\gamma_{n}$.

Let $\pi: \mathbb{R}_{\alpha} \rightarrow \mathbb{R}$ be the map that 'forgets' the ' + ' or ' - '. For each $x \in \mathbb{R}_{\alpha}$, let $[x]$ be the greatest integer less than or equal to $x$ - with the convention that $\left[n^{+}\right]=n$ and $\left[n^{-}\right]=n-1$ for $n \in \mathbb{Z}$. Also $\{x\}$ will denote the fractional part of $x$ : $\{x\}=\pi(x)-[\pi(x)]$.

1.9. Definition. For $\alpha=\left[0 ; a_{1}, a_{2}, a_{3}, \ldots\right]$ let

$$
\begin{array}{ll}
\alpha_{0}=1 & \alpha_{1}=\alpha \\
\alpha_{2}=1-a_{1} \alpha=\alpha\left\{\alpha^{-1}\right\} & \alpha_{3}=\alpha_{1}-a_{2} \alpha_{2}=\alpha_{2}\left\{\alpha_{1} \alpha_{2}^{-1}\right\} \\
\quad \vdots & \\
\alpha_{n+1}=\alpha_{n-1}-a_{n} \alpha_{n}=\alpha_{n}\left\{\alpha_{n-1} \alpha_{n}^{-1}\right\} &
\end{array}
$$

and for $\beta \in \mathbb{R}_{\alpha}$, let $\beta_{1}=\beta-[\beta]$ and

$$
x_{1}=\left[\beta_{1} / \alpha_{1}\right] \quad \text { and } \quad \beta_{2}= \begin{cases}\left(1+x_{1}\right) \alpha_{1}-\beta_{1} & \text { if } x_{1}<a_{1} \\ \alpha_{0}-\beta_{1} & \text { if } x_{1}=a_{1} .\end{cases}
$$

If $\beta_{1}, \beta_{2}, \ldots, \beta_{n}$ and $x_{1}, x_{2}, x_{3}, \ldots, x_{n-1}$ have been constructed, let

$$
x_{n}=\left[\beta_{n} / \alpha_{n}\right] \quad \text { and } \quad \beta_{n+1}= \begin{cases}\left(1+x_{n}\right) \alpha_{n}-\beta_{n} & \text { if } x_{n}<a_{n} \\ \alpha_{n-1}-\beta_{n} & \text { if } x_{n}=a_{n} .\end{cases}
$$

Let $\varphi: \mathbb{R}_{\alpha} \rightarrow X_{\alpha}$ be the map $\varphi(\beta)=\left(x_{i}\right)_{i=1}^{\infty}$.

We shall show in Theorem B that if we start with a line $\mathrm{L}: y=\alpha x+\beta$, and let $\mathrm{T}$ be the corresponding coding sequence, and $t_{1}=b_{0}$, the intersection of $\mathrm{L}$ with the $y$-axis, then the coding sequence associated with $(\mathrm{T}, \mathrm{t})$ is $\varphi(\beta)=\left(x_{1}, x_{2}, x_{3}, \ldots\right) \in$ $X_{\alpha}$.

1.10. Remark. Let us recall two results from [JAM]. Let $S_{\mathbb{N} \alpha}^{1}$ be the Cantor set obtained by cutting the circle $S^{1}$ along the forward orbit of 0 under the action of rotation by the angle $2 \pi \alpha$. Writing $S^{1}$ as $[0,1]$ with 0 and 1 identified and the group law as addition mod $1, S_{\mathbb{N} \alpha}^{1}$ becomes the interval $\left[0^{+}, 1^{-}\right] \subseteq \mathbb{R}_{\alpha}$. Theorem 3.6 of [JAM] showed that $\varphi:\left[0^{+}, 1^{-}\right] \rightarrow X_{\alpha}$ is a homeomorphism and that (by Theorem 3.8 and Corollary 3.9) $\varphi(x)$ is tail equivalent to $-\alpha, 0^{+}$, or $0^{-}$if and only if $x=-m \alpha, x=n \alpha^{+}$, or $x=n \alpha^{-}$respectively for $m>0$ and $n \geq 0$. This implies that, given a coding sequence $\left(x_{i}\right)$, we can use the formulas of 1.9 to construct a line which gives the coding sequence $\left(x_{i}\right)$. In the paragraph below we give a graphical interpretation of this.

1.11. Remark. In Figure 1 we illustrate the expansion of $\beta$, i.e. the map $[0,1] \ni$ $\beta \mapsto\left(x_{i}\right) \in X_{\alpha}$ when $\alpha=[0 ; 3,1,3,1, \ldots] \approx 0.26$. In the figure the interval $[0,1]$ has been cut at the points $n \alpha(\bmod 1)$ for integers $n \geq 0$. In the first row we have made a partition of $[0,1]$ using the points $\left\{0, \alpha, 2 \alpha, \ldots, a_{1} \alpha\right\}$. In the second row we have refined the partition by adding the points $\left\{\left(1+a_{1}\right) \alpha, \ldots, a_{1}\left(1+a_{2}\right) \alpha\right\}$. The rule for the $i$-th row is to add the points $\left\{\left(q_{i-2}+q_{i-1}\right) \alpha, \ldots,\left(q_{i}+q_{i-1}-1\right) \alpha\right\}$, 


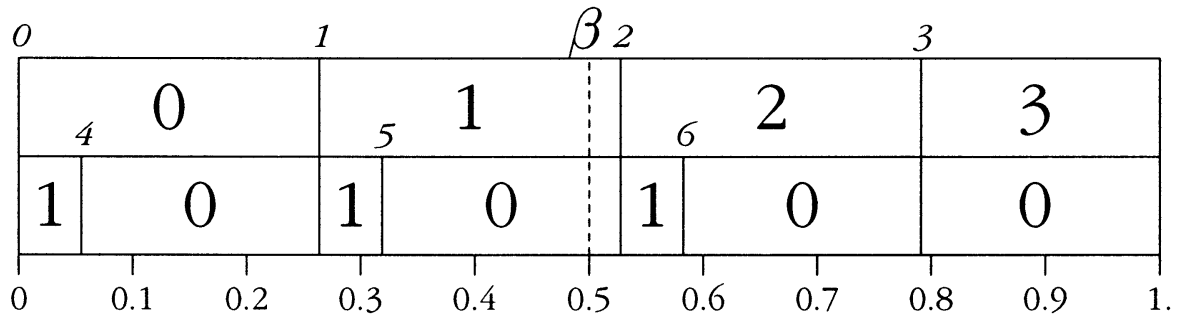

FIGURE 1.

where $q_{0}=1, q_{1}=a_{1}$, and $q_{i+1}=a_{i+1} q_{i}+q_{i-1}$ are the usual denominators of the convergents in the continued fraction expansion of $\alpha$.

We can also view the partition as being constructed by a 'paving'. We begin by 'paving' the interval $[0,1]$ from left to right by interval of length $\alpha$. We can get in $a_{1}$ of these intervals, and there is a remainder of length $\alpha_{2}$. So in the top row the intervals are of length $\alpha_{1}=\alpha$, except for the last which is of length $\alpha_{2}=1-a_{1} \alpha_{1}$. To obtain the second row we 'pave' from right to left each of the intervals on the first row with intervals of length $\alpha_{2}$. We can fit in $a_{2}$ intervals of length $\alpha_{2}$ in each interval of length $\alpha$. Thus on the second row there are intervals of length $\alpha_{2}$ and remainders of length $\alpha_{3}$. To construct the $i$-th row we 'pave' each interval of the $(i-1)$-st row with intervals of length $\alpha_{i}$. When $i$ is odd we pave from left to right and when $i$ is even we pave from right to left.

The numbers $1,2,3, \ldots, 6$ indicate the multiples of $\alpha$ (modulo 1 ). In this example $\alpha \approx 0.26$, so $4 \alpha(\bmod 1)$ is a little to the right of 0 . On the top row we calculate $x_{1}$. Thus if $\beta \in[i \alpha,(i+1) \alpha]$ then $x_{i}=i$. On the bottom row we calculate $x_{2}$. In this example $x_{2} \in\{0,1\}$. If $x_{1}=a_{1}$ then $x_{2}$ can only be 0 . Otherwise we count the number of intervals from the right wall of the containing interval on the first row. Thus when $\beta=0.5$ we have $x_{1}=1$ and $x_{2}=0$.

1.12. Definition. Given two cutting sequences $T$ and $T^{\prime}$ in $\mathcal{T}_{\alpha}$ and chosen letters $t$ and $t^{\prime}$ in $T$ and $T^{\prime}$ respectively, we say that $(T, t)$ and $\left(T^{\prime}, t^{\prime}\right)$ are translation equivalent if there is $\tilde{\mathrm{t}}^{\prime}$ in $\mathrm{T}^{\prime}$ such that $(\mathrm{T}, \mathrm{t})=\left(\mathrm{T}^{\prime}, \tilde{\mathrm{t}}^{\prime}\right)$.

Recall from 1.2 that for each $\alpha$ there is a pair of singular cutting sequences $\mathrm{T}^{+}$ and $\mathrm{T}^{-}$:

$$
\begin{aligned}
\mathrm{T}^{+} & =\ldots \mathrm{bbb} \mathrm{ab} b b b \\
\mathrm{~T}^{-} & =\ldots \mathrm{bbb} \mathrm{ba} b b b \ldots
\end{aligned}
$$

These sequences were called conjugate because reflection about the centre of the box takes one to the other. Our invariant cannot separate these two sequences. If we choose a letter to the left of the box in either sequence we get a coding sequence tail equivalent to $-\alpha$; if we choose a letter to the right of the box we get a coding sequence tail equivalent to $0^{+}$or $0^{-}$respectively. Therefore on our space $\mathcal{T}_{\alpha}^{\bullet}$ of pointed cutting sequence with slope $\alpha$ we put the equivalence relation $\approx$ generated by translation and conjugation.

To conclude this introduction we present the main results of this paper. The numbers following the lettering of the theorems will direct the reader to the section containing the proof. Our results were inspired by and are analogous to the statements 10.5.9-10.5.14 in [GS] for Penrose tilings. 
Theorem A (3.1). Let $T$ be a non-singular cutting sequence and $t$ and $t^{\prime}$ be tiles in T. Let $x$ and $x^{\prime}$ in $X_{\alpha}$ be the corresponding coding sequences. Then $x$ and $x^{\prime}$ are tail equivalent.

Let $T$ be a singular cutting sequence and $t$ a tile in $T$. Then the coding sequence of $t$ is tail equivalent to one of $-\alpha, 0^{+}$, or $0^{-}$.

Theorem A shows that except for the singular cutting sequences, the tail equivalence in $X_{\alpha}$ of any coding sequence is an invariant of $\mathrm{T}$ under translation. For the singular cutting sequences there are three possible tail equivalence classes.

Theorem B (3.2). Let $\beta \in \mathbb{R}_{\alpha}$, and let $L$ be the line $y=\alpha x+\beta$. Let $T$ be the corresponding cutting sequence, let $t$ be the tile $b_{0}$ (as described in 1.2), and let $x \in X_{\alpha}$ be the corresponding coding sequence. Then $x=\varphi(\beta)$, where $\varphi$ is constructed in 1.9 In particular, the cutting sequence $T$ is singular if and only if $L$ passes through a point of $\mathbb{Z}^{2}$.

Theorem C (3.4). Let $T$ be a cutting sequence, $t$ a tile in $T$, and $x$ the coding sequence of $(T, t)$. Then the Smith sequence $\left\{T_{i}\right\}_{i=1}^{\infty}$ of $x$ equals the inflation sequence $\left\{W_{i}\right\}_{i=1}^{\infty}$ of $(T, t)$ i.e. $W_{i}=T_{i}$ for $i=1,2,3, \ldots$

Theorem D (3.5). Let $\sim$ be the equivalence relation on $X_{\alpha}$ generated by tail equivalence, and let $-\alpha \sim 0^{+} \sim 0^{-}$. Let $\left(T_{1}, t_{1}\right)$ and $\left(T_{2}, t_{2}\right)$ be in $\mathcal{T}_{\alpha}^{\bullet}$, and $x_{1}$ and $x_{2}$ in $X_{\alpha}$ the corresponding coding sequences. Then $x_{1} \sim x_{2}$ if and only if $T_{1} \approx T_{2}$.

\section{Preliminaries}

Before proving our main results we recall some elementary lemmas. Let $0<\alpha<$ 1 be irrational and $\beta \in \mathbb{R}_{\alpha}$. Let $\mathrm{L}$ be the line $y=\alpha x+\beta$. Let $\mathrm{T}$ be the sequence obtained by the cutting method (1.2).

Lemma 2.1. Between adjacent a's there are either $\left[\alpha^{-1}\right]$ or $1+\left[\alpha^{-1}\right] b^{\prime}$ s. There are $1+\left[\alpha^{-1}\right]$ b's between $a_{i}$ and $a_{i+1}$ if and only if $\left\{i+1-\frac{\beta}{\alpha}\right\} \leq\left\{\frac{1}{2}\right\}$, unless $T=T^{-}$and $\left\{i-\frac{\beta}{\alpha}\right\} \in \mathbb{Z}$, in which case there are $\left[\alpha^{-1}\right] b$ 's between $a_{i}$ and $a_{i+1}$, or $T=T^{+}$and $i+1-\frac{\beta}{\alpha} \in \mathbb{Z}$, in which case there are $\left[\alpha^{-1}\right]$ b'a between $a_{i}$ and $a_{i+1}$.

Proof. Suppose neither $i-\frac{\beta}{\alpha}$ nor $i+1-\frac{\beta}{\alpha}$ is an integer. Then the number of b's between $a_{i}$ and $a_{i+1}$ is the number of integers in the interval $\left[i-\frac{\beta}{\alpha}, i+1-\frac{\beta}{\alpha}\right]$. This interval contains $\left[i+1-\frac{\beta}{\alpha}\right]-\left[i-\frac{\beta}{\alpha}\right]=\left[\alpha^{-1}\right]+\left[\left\{i-\frac{\beta}{\alpha}\right\}+\left\{\alpha^{-1}\right\}\right]$ integers. Thus there are either $\left[\alpha^{-1}\right]$ or $1+\left[\alpha^{-1}\right]$ b's between $\mathrm{a}_{i}$ and $\mathrm{a}_{i+1}$. The interval $\left[i-\frac{\beta}{\alpha}, i+1-\frac{\beta}{\alpha}\right]$ contains $1+\left[\alpha^{-1}\right]$ integers $\Longleftrightarrow\left[\left\{i-\frac{\beta}{\alpha}\right\}+\left\{\alpha^{-1}\right\}\right]=1 \Longleftrightarrow$ $\left\{i-\frac{\beta}{\alpha}\right\}+\left\{\alpha^{-1}\right\}>1 \Longleftrightarrow\left\{i+1-\frac{\beta}{\alpha}\right\}=\left\{\left\{i-\frac{\beta}{\alpha}\right\}+\left\{\alpha^{-1}\right\}\right\}<\left\{\alpha^{-1}\right\}$.

Suppose $i-\frac{\beta}{\alpha} \in \mathbb{Z}$. Then $\mathrm{b}_{i-\frac{\beta}{\alpha}}$ is between $\mathrm{a}_{i}$ and $\mathrm{a}_{i+1}$ only when $\mathrm{T}=\mathrm{T}^{+}$. So when $\mathrm{T}=\mathrm{T}^{+}$, we have $\left\{i+1-\frac{\beta}{\alpha}\right\}=\left\{\frac{1}{\alpha}\right\}$, and there are $1+\left[\alpha^{-1}\right] \mathrm{b}$ 's between $\mathrm{a}_{i}$ and $\mathrm{a}_{i+1}$, and when $\mathrm{T}=\mathrm{T}^{-}$we have $\left[\alpha^{-1}\right]$ b's between $\mathrm{a}_{i}$ and $\mathrm{a}_{i+1}$.

Suppose $i+1-\frac{\beta}{\alpha} \in \mathbb{Z}$. Then $\mathrm{b}_{i+1-\frac{\beta}{\alpha}}$ is between $\mathrm{a}_{i}$ and $\mathrm{a}_{i+1}$ only when $\mathrm{T}=\mathrm{T}^{-}$. So when $\mathrm{T}=\mathrm{T}^{-}$and $i+1-\frac{\beta}{\alpha} \in \mathbb{Z}$ we have $\left\{i+1-\frac{\beta}{\alpha}\right\}=0<\left\{\frac{1}{\alpha}\right\}$, and there are $1+\left[\alpha^{-1}\right]$ b's between $\mathrm{a}_{i}$ and $\mathrm{a}_{i+1}$, and when $\mathrm{T}=\mathrm{T}^{+}$, we have $\left[\alpha^{-1}\right]$ b's between $\mathrm{a}_{i}$ and $\mathrm{a}_{i+1}$. 
Lemma 2.2. Let $k\left(b_{i}\right)$ be the number of b's between $b_{i}$ and the first a to the left of $b_{i}$. Then $k\left(b_{i}\right)=\left[\{i \alpha+\beta\} \alpha^{-1}\right]$ except in the cases

(i) $T=T$ and $i \alpha+\beta \in \mathbb{Z}$, in which case $k\left(b_{i}\right)=\left[\alpha^{-1}\right]$, and

(ii) $T=T,[i \alpha+\beta]-\frac{\beta}{\alpha} \in \mathbb{Z}$ but $i \alpha+\beta \notin \mathbb{Z}$, in which case

$$
k\left(b_{i}\right)=\{i \alpha+\beta\} \alpha^{-1}-1 .
$$

Proof. Suppose neither $i \alpha+\beta$ nor $[i \alpha+\beta]-\frac{\beta}{\alpha}$ is an integer. The first a to the left of $\mathrm{b}_{i}$ is $\mathrm{a}_{[i \alpha+\beta]}$. The first $\mathrm{b}$ following $\mathrm{a}_{[i \alpha+\beta]}$ is $\mathrm{b}_{1+\left[[i \alpha+\beta]-\frac{\beta}{\alpha}\right]}$. Thus the number of b's between $\mathrm{b}_{i}$ and $\mathrm{a}_{[i \alpha+\beta]}$ is $i-\left(1+\left[[i \alpha+\beta]-\frac{\beta}{\alpha}\right]\right)=\left[\{i \alpha+\beta\} \alpha^{-1}\right]$.

Suppose $i \alpha+\beta \in \mathbb{Z}$. When $\mathrm{T}=\mathrm{T}^{+}$there are $0 \mathrm{~b}$ 's between $\mathrm{b}_{i}$ and $\mathrm{a}_{i \alpha+\beta}$, and the first a to the left of $b_{i}$. When $T=T^{-}$the first a to the left is $a_{i \alpha+\beta-1}$, the first $\mathrm{b}$ following $\mathrm{a}_{i \alpha+\beta-1}$ is $\mathrm{b}_{1+\left[i \alpha+\beta-1-\frac{\beta}{\alpha}\right]}=\mathrm{b}_{i-\left[\alpha^{-1}\right]}$. Thus there are $i-\left(i-\left[\alpha^{-1}\right]\right)$ b's between $\mathrm{b}_{i}$ and the first a to its left.

Suppose $[i \alpha+\beta]-\frac{\beta}{\alpha} \in \mathbb{Z}$ but $i \alpha+\beta \notin \mathbb{Z}$. When $\mathrm{T}=\mathrm{T}^{+}$, the first a to the left of $\mathrm{b}_{i}$ is $\mathrm{a}_{[i \alpha+\beta]}$ and the first $\mathrm{b}$ following $\mathrm{a}_{[i \alpha+\beta]}$ is $\mathrm{b}_{[i \alpha+\beta]-\frac{\beta}{\alpha}}$; thus the number of b's between $\mathrm{b}_{i}$ and $\mathrm{a}_{[i \alpha+\beta]}$ is $i-[i \alpha+\beta]-\frac{\beta}{\alpha}=\{i \alpha+\beta\} \alpha^{-1}$. When $\mathrm{T}=\mathrm{T}^{-}$, the first a to the left of $\mathrm{b}_{i}$ is again $\mathrm{a}_{[i \alpha+\beta]}$, but the first $\mathrm{b}$ following $\mathrm{a}_{[i \alpha+\beta]}$ is now $\mathrm{b}_{1+[i \alpha+\beta]-\frac{\beta}{\alpha}}$; thus the number of $\mathrm{b}$ 's between $\mathrm{a}_{[i \alpha+\beta]}$ and $\mathrm{b}_{i}$ is $\{i \alpha+\beta\} \alpha^{-1}-1$.

The next proposition shows how to keep track of $b_{0}$ under composition.

Proposition 2.3. Let $L$ be the line $y=\alpha x+\beta, T$ the sequence obtained from $L$ by the cutting method, and let $T^{\prime}$ be the sequence obtained from the the line $y=\left\{\alpha^{-1}\right\} x-\beta \alpha^{-1}$ by the cutting method. If $L$ passes through a point of $\mathbb{Z}^{2}$ and $T=T^{+}$let $T^{\prime}=T^{\prime-}$, and if $T=T^{-}$let $T^{\prime}=T^{\prime+}$.

For $T \neq T^{-}$we define a map from $T$ to $T^{\prime}$ by sending

$$
\begin{array}{ll}
T \ni a_{i} & \mapsto b_{i} \in T^{\prime} \\
T \ni b_{i} & \mapsto \begin{cases}b_{[i \alpha+\beta]} \in T^{\prime} & \text { if }\{i \alpha+\beta\} \alpha^{-1}<\left[\alpha^{-1}\right] \\
a_{i-\left[\alpha^{-1}\right](1+[i \alpha+\beta])} \in T^{\prime} & \text { if }\{i \alpha+\beta\} \alpha^{-1} \geq\left[\alpha^{-1}\right] .\end{cases}
\end{array}
$$

For $T=T^{-}$the map is given by

$$
\begin{aligned}
T \ni a_{i} & \mapsto b_{i} \in T^{\prime} \\
T \ni b_{i} & \mapsto \begin{cases}b_{[i \alpha+\beta]} \in T^{\prime} & \text { if }\{i \alpha+\beta\} \alpha^{-1}<\left[\alpha^{-1}\right] \\
a_{i-\left[\alpha^{-1}\right](1+[i \alpha+\beta])} \in T^{\prime} & \text { and } i \alpha+\beta \notin \mathbb{Z}\{i \alpha+\beta\} \alpha^{-1} \geq\left[\alpha^{-1}\right] \\
a_{i-\left[\alpha^{-1}\right](i \alpha+\beta)} \in T^{\prime} & \text { if } i \alpha+\beta \in \mathbb{Z} .\end{cases}
\end{aligned}
$$

Then $T^{\prime}$ is the sequence obtained from $T$ by composition, and the map above from $T$ to $T^{\prime}$ is the composition map.

Proof. We must show that each word $a b \cdots b$ in $\mathrm{T}$ with $\left[\alpha^{-1}\right]$ b's gets mapped to a $\mathrm{b}$ in $\mathrm{T}^{\prime}$, and each word $\mathrm{ab} \cdots \mathrm{b}$ in $\mathrm{T}$ with $1+\left[\alpha^{-1}\right]$ b's gets mapped to ba in $\mathrm{T}^{\prime}$.

To simplify the calculations let $\mathrm{L}$ denote the original line $y=\alpha x+\beta$ and $\widetilde{\mathrm{L}}$ denote the line $y=\left\{\alpha^{-1}\right\}^{-1} x+\beta \alpha^{-1}\left\{\alpha^{-1}\right\}^{-1}$. Note that $\widetilde{\mathrm{L}}$ is the reflection in $y=x$ of the line $y=\left\{\alpha^{-1}\right\} x-\beta \alpha^{-1}$. Let us denote by $\mathrm{A}_{i}$ the intersection of $\widetilde{\mathrm{L}}$ with $y=i$ 
and $\mathrm{B}_{i}$ the intersection of $\widetilde{\mathrm{L}}$ with $x=i$. Under reflection in the line $y=x, \mathrm{~A}_{i}$ gets mapped to $\mathrm{b}_{i}$ and $\mathrm{B}_{i}$ to $\mathrm{a}_{i}$. Under this transformation our map then is

$$
\begin{aligned}
\mathrm{T} \ni \mathrm{a}_{i} & \mapsto \mathrm{A}_{i} \\
\mathrm{~T} \ni \mathrm{b}_{i} & \mapsto \begin{cases}\mathrm{A}_{[i \alpha+\beta]} & \text { if }\{i \alpha+\beta\} \alpha^{-1}<\left[\alpha^{-1}\right] \\
\mathrm{B}_{i-\left[\alpha^{-1}\right](1+[i \alpha+\beta])} & \text { if }\{i \alpha+\beta\} \alpha^{-1} \geq\left[\alpha^{-1}\right]\end{cases}
\end{aligned}
$$

and when $\mathrm{T}=\mathrm{T}^{-}$

$$
\begin{aligned}
& \mathrm{T} \ni \mathrm{a}_{i} \mapsto \mathrm{A}_{i} \\
& \mathrm{~T} \ni \mathrm{b}_{i} \mapsto \begin{cases}\mathrm{A}_{[i \alpha+\beta]} & \text { if }\{i \alpha+\beta\} \alpha^{-1}<\left[\alpha^{-1}\right] \\
\mathrm{B}_{i-\left[\alpha^{-1}\right](1+[i \alpha+\beta])} & \text { if }\{i \alpha+\beta\} \alpha^{-1} \geq\left[\alpha^{-1}\right] \\
\mathrm{B}_{i-\left[\alpha^{-1}\right](i \alpha+\beta)} & \text { if } i \alpha+\beta \in \mathbb{Z} .\end{cases}
\end{aligned}
$$

To start with, let us suppose that $T \neq T^{-}$. For each $b_{i}$ the first a to the left is $\mathrm{a}_{[i \alpha+\beta]}$, and thus $\mathrm{b}_{i}$ and $\mathrm{a}_{[i \alpha+\beta]}$ get mapped to the same letter: $\mathrm{A}_{[i \alpha+\beta]}$, unless $k\left(\mathrm{~b}_{i}\right)=\left[\alpha^{-1}\right]$, i.e. $\{i \alpha+\beta\} \alpha^{-1} \geq\left[\alpha^{-1}\right]$. So we must check that

(†) There are $1+\left[\alpha^{-1}\right]$ b's between $a_{[i \alpha+\beta]}$ and $a_{1+[i \alpha+\beta]}$ if and only if there is a $B$ between $A_{[i \alpha+\beta]}$ and $A_{1+[i \alpha+\beta]}$.

Now for any $j$

$$
\begin{aligned}
& \text { there are } 1+\left[\alpha^{-1}\right] \mathbf{b}^{\prime} s \text { between } \mathrm{a}_{j} \text { and } \mathrm{a}_{j+1} \\
& \Longleftrightarrow\left\{j+1-\frac{\beta}{\alpha}\right\}<\left\{\frac{1}{\alpha}\right\} \\
& \Longleftrightarrow\left\{\left\{\alpha^{-1}\right\}(j+1)-\beta \alpha^{-1}\right\}=\left\{\alpha^{-1}(j+1)-\beta \alpha^{-1}-\left[\alpha^{-1}\right](j+1)\right\} \\
& =\left\{j+1-\frac{\beta}{\alpha}\right\}<\left\{\frac{1}{\alpha}\right\} \\
& \Longleftrightarrow\left\{\alpha^{-1}\right\}(j+1)-\beta \alpha^{-1}-\left\{\left\{\alpha^{-1}\right\}(j+1)-\beta \alpha^{-1}\right\} \\
& >\left\{\alpha^{-1}\right\} j-\beta \alpha^{-1} \\
& \Longleftrightarrow\left[\left\{\alpha^{-1}\right\}(j+1)-\beta \alpha^{-1}\right]>\left\{\alpha^{-1}\right\} j-\beta \alpha^{-1} \\
& \Longleftrightarrow \text { there is a } \mathrm{B} \text { between } \mathrm{A}_{j} \text { and } \mathrm{A} j+1 \text {. }
\end{aligned}
$$

Letting $j=[i \alpha+\beta]$, we have $(\dagger)$. This equivalence holds even when $j+1-\frac{\beta}{\alpha} \in \mathbb{Z}$ (and $\mathrm{T}=\mathrm{T}^{+}$), for then $\mathrm{a}_{j+1}$ is at a point of $\mathbb{Z}^{2}$ and there will be only $\left[\alpha^{-1}\right]$ b's between $\mathrm{a}_{j}$ and $\mathrm{a}_{j+1}$, while there will not be a $\mathrm{B}$ between $\mathrm{A}_{j}$ and $\mathrm{A}_{j+1}$ in this case because $A_{j+1}$ will also be at a point of $\mathbb{Z}^{2}$ and so $A_{j+1}$ will be to the left of the coinciding $B$ (since $T^{\prime}=T^{\prime-}$ ).

When there is a $B$ between $A_{[i \alpha+\beta]}$ and $A_{1+[i \alpha+\beta]}$ it will be

$$
\mathrm{B}_{1+\left[\frac{[i \alpha+\beta]-\beta \alpha^{-1}\left\{\alpha^{-1}\right\}^{-1}}{\left\{\alpha^{-1}\right\}^{-1}}\right]}=\mathrm{B}_{1+\left[\left\{\alpha^{-1}\right\}[i \alpha+\beta]-\beta \alpha^{-1}\right]}=\mathrm{B}_{i-\left[\alpha^{-1}\right](1+[i \alpha+\beta])} \text {. }
$$

Now suppose $T=T^{-}$. The only additional complication is when $b_{i}$ is at a point of $\mathbb{Z}^{2}$. Since $T=T^{-}$, this can only occur when $b_{i}$ is the last $\mathrm{b}$ in a block of $1+\left[\alpha^{-1}\right]$. So suppose $i \alpha+\beta \in \mathbb{Z}$; then $\mathrm{b}_{i}$ and $\mathrm{a}_{i \alpha+\beta}$ coincide at $(i, i \alpha+\beta) \in \mathbb{Z}^{2}$ and $\mathrm{b}_{i}$ is to the left of $\mathrm{a}_{i \alpha+\beta}$. $\mathrm{a}_{i \alpha+\beta}$ gets sent to $\mathrm{A}_{i \alpha+\beta}$, which will be at the point $\left(i-\left[\alpha^{-1}\right](i \alpha+\beta), i \alpha+\beta\right)$ of $\mathbb{Z}^{2}$ on the line $\widetilde{L}$. So $\mathrm{B}_{i-\left[\alpha^{-1}\right][i \alpha+\beta]}$ will be to the left of 
$\mathrm{A}_{i \alpha+\beta}$, because $\mathrm{a}_{i-\left[\alpha^{-1}\right](i \alpha+\beta)}$ will be to the left of $\mathrm{b}_{i \alpha+\beta}$ in $\mathrm{T}^{\prime}=\mathrm{T}^{\prime+}$. Hence there will be a $\mathrm{B}$ between $\mathrm{A}_{i \alpha+\beta-1}$, and let $\mathrm{A}_{i \alpha+\beta}$.

\section{The Proof of Theorems A, B, C, And D}

Theorem 3.1 (Theorem A). Let $T$ be a non-singular cutting sequence and $t$ and $t^{\prime}$ be tiles in T. Let $x$ and $x^{\prime}$ in $X_{\alpha}$ be the corresponding coding sequences. Then $x$ and $x^{\prime}$ are tail equivalent.

Let $T$ be a singular cutting sequence, and let $t$ a tile in $T$. Then the coding sequence of $t$ is tail equivalent to one of $-\alpha, 0^{+}$, or $0^{-}$.

Proof. Suppose that $\mathrm{T}$ is non-singular. Then $\mathrm{T}=\bigcup_{i=0}^{\infty} W_{i}$, where $\left\{W_{i}\right\}$ is the inflation sequence of some letter $\widetilde{\mathrm{t}}$ in $\mathrm{T}$ (cf. section 1.7). Then for all $i$ larger than some $i_{0}, \mathrm{t}$ and $\mathrm{t}^{\prime}$ are in the same segment $W_{i}$. For each letter $\mathrm{s}$ in $W_{i}$ the $i$-th element of the coding sequence of $\mathrm{s}$ is the same as the $i$-th element of the coding sequence of $\widetilde{\mathrm{t}}$. Hence from the $i$-th element on the coding sequences of $\mathrm{t}$ and $\mathrm{t}^{\prime}$ agree.

Suppose that $\mathrm{T}$ is singular and $\mathrm{t} \in \mathrm{T}$. Let $W_{0}=\{\mathrm{t}\} \subseteq W_{1} \subseteq W_{2} \cdots$ be the inflation sequence of $\mathrm{t}$. Then $\bigcup_{i=0}^{\infty} W_{i}$ is a connected infinite proper subset of $\mathrm{T}$ and is thus a right or left half line.

Case 1: $\bigcup_{i=0}^{\infty} W_{i}$ is a left half line. Let $\mathrm{t}^{-}$be the rightmost letter of $\bigcup_{i=0}^{\infty} W_{i}$. We claim that $\mathrm{t}^{-}$is $\mathrm{a} \mathrm{b}$ and that to the left of $\mathrm{t}^{-}$is the segment $\underbrace{\mathrm{b} \cdots \mathrm{b}}_{a_{1}-1}$, where $\alpha=\left[0 ; a_{1}, a_{2}, a_{3}, \ldots\right]$ is the slope of $\mathrm{T}$. Indeed, $\mathrm{t}^{-}$cannot be an a, as it would be grouped with $\mathrm{a} b$ to the right (and thus outside of $\bigcup_{i=0}^{\infty} W_{i}$ ) on the first composition. Thus $\mathrm{t}^{-}$is a $\mathrm{b}$. If there are fewer than $a_{1}-2$ b's between $\mathrm{t}^{-}$and the first a to the left, then $a b$ to the right of $t^{-}$would be grouped with $t^{-}$on the first composition, which is again impossible.

When we perform composition on $\mathrm{T}, \mathrm{t}^{-}$gets grouped into a letter which is also the rightmost letter in a half line, and by the same argument as above this letter is $\mathrm{a} b$ and to its left is the segment $\underbrace{\mathrm{b} \cdots \mathrm{b}}_{a_{2}-1}$. This is repeated under successive compositions, and hence the coding sequence of $\mathrm{t}^{-}$is $\left(a_{1}-1, a_{2}-1, a_{3}-1, \ldots\right)=-\alpha$. If $\mathrm{t}$ is any letter in $\bigcup_{i=0}^{\infty} W_{i}$, then eventually $\mathrm{t}$ and $\mathrm{t}^{-}$are in some $W_{i}$. From this point on the coding sequence of $\mathrm{t}^{-}$and $\mathrm{t}^{-}$are the same, and thus the coding sequence of $\mathrm{t}$ is tail equivalent to $-\alpha$, and $\bigcup_{i=1}^{\infty} W_{i}=\mathrm{T}_{-\alpha}$ (see 1.6).

Case 2: Now let us suppose that $\bigcup_{i=0}^{\infty} W_{i}$ is a right half line. This time, let $\mathrm{t}^{+}$ be the leftmost letter of $\bigcup_{i=0}^{\infty} W_{i}$, and let $\mathrm{t}_{1}=\mathrm{t}^{+}, \mathrm{t}_{2}, \ldots$ be the sequence of letters obtained by following $\mathrm{t}^{+}$through successive compositions. From Case 1 we see that $\mathrm{t}_{i}$ will always have $\underbrace{\mathrm{b} \cdots \mathrm{b}}_{a_{i}}$ to its immediate left. Thus, if $\mathrm{t}^{+}$is an $\mathrm{a}$, then $x_{1}=0$ and $\mathrm{t}_{2}$ is a $\mathrm{b}$. Then $x_{2}=a_{2}$ and $\mathrm{t}_{3}$ is an a again, and the process repeats. Hence $\left(x_{i}\right)=$ $\left(0, a_{2}, 0, a_{4}, \ldots\right)=0^{+}$, and $\bigcup_{i=1}^{\infty} W_{i}=\mathrm{T}_{0^{+}}$(see 1.6). Similarly, if $\mathrm{t}^{+}$is a $\mathrm{b}$, then $\mathrm{t}_{2}$ is an a and $t_{3}$ is a $\mathrm{b}$. In this case the coding sequence $\left(x_{i}\right)=\left(a_{1}, 0, a_{3}, 0, \ldots\right)=0^{-}$, and $\bigcup_{i=1}^{\infty} W_{i}=\mathrm{T}_{0^{-}}$(see[1.6).

If $\mathrm{t}$ is any letter in $\bigcup_{i=0}^{\infty} W_{i}$, then $\mathrm{t}$ and $\mathrm{t}^{+}$are eventually in some $W_{i}$, and thus from $i$ onwards their coding sequences will be equal. Hence the coding sequence for $\mathrm{t}$ will be tail equivalent to either $0^{+}$or $0^{-}$. 
Theorem 3.2 (Theorem B). Let $\beta \in \mathbb{R}_{\alpha}$, and let $L$ be the line $y=\alpha x+\beta$. Let $T$ be the corresponding cutting sequence, let $t$ be the tile $b_{0}$ (as described in 1.2), and let $x \in X_{\alpha}$ be the corresponding coding sequence. Then $x=\varphi(\beta)$, where $\varphi$ is constructed in [1.9. In particular, the cutting sequence $T$ is singular if and only if $L$ passes through a point of $\mathbb{Z}^{2}$.

Proof. Let $\varphi(\beta)=\left(x_{1}, x_{2}, x_{3}, \ldots\right)$. Both $\varphi(\beta)$ and the coding sequence for $\mathrm{b}_{0}$ are unchanged by replacing $\beta$ by $\beta-[\beta]$, so we shall suppose that $0 \leq \beta \leq 1$.

We shall show that if $L^{\prime \prime}$ is the line $y=\left\{\alpha^{-1}\right\} x+\beta_{2}$, where $\beta_{2}=1-\frac{\beta}{\alpha}$ if $\beta \alpha^{-1} \geq\left[\alpha^{-1}\right]$ or $\beta_{2}=\left(1+x_{1}\right) \alpha-\frac{\beta}{\alpha}$ if $\beta \alpha^{-1}<\left[\alpha^{-1}\right]$, and $\mathrm{T}^{\prime \prime}$ is the corresponding cutting sequence, then $T^{\prime \prime}$ is the composition of $T$, and $b_{0}$ in $T$ gets sent to either $\mathrm{b}_{0}$ in $\mathrm{T}^{\prime \prime}$ when $\beta \alpha^{-1}<\left[\alpha^{-1}\right]$, or to $\mathrm{a}_{0}$ in $\mathrm{T}^{\prime \prime}$ when $\beta \alpha^{-1} \geq\left[\alpha^{-1}\right]$.

When $\beta \alpha^{-1} \geq\left[\alpha^{-1}\right], \mathrm{b}_{0}$ is the first $\mathrm{b}$ in $\mathrm{T}^{\prime \prime}$ to the right of $\mathrm{a}_{0}$. So $\mathrm{a}_{0}$, and $\mathrm{b}_{0}$ get grouped under composition and have the same coding sequence. Thus the theorem follows by induction if we can prove the claim made above about $L^{\prime \prime}$ and $T^{\prime \prime}$.

Let us recall some notation from Proposition 2.3. Let $\mathrm{L}^{\prime}$ be the line $y=\left\{\alpha^{-1}\right\} x-$ $\beta \alpha^{-1}$ and $\mathrm{T}^{\prime}$ its cutting sequence. Applying Proposition 2.3 to the case $i=0$ we see that $\mathrm{b}_{0}$ in $\mathrm{T}$ gets sent either to $\mathrm{b}_{0}$ in $\mathrm{T}^{\prime}$ when $\beta \alpha^{-1}<\left[\alpha^{-1}\right]$, or to $\mathrm{a}_{-\left[\alpha^{-1}\right]}$ in $\mathrm{T}^{\prime}$ when $\beta \alpha^{-1} \geq\left[\alpha^{-1}\right]$.

Case 1: $\beta \alpha^{-1}<\left[\alpha^{-1}\right]$. Then $\beta_{2}=1+x_{1}-\left\{\beta \alpha^{-1}\right\}=-\beta \alpha^{-1}+1+x_{1}+\left[\beta \alpha^{-1}\right]$. Thus $\mathrm{L}^{\prime \prime}$ is just $\mathrm{L}^{\prime}$ raised by $1+x_{1}+\left[\beta \alpha^{-1}\right]$. This means that $\mathrm{T}^{\prime}$ and $\mathrm{T}^{\prime \prime}$ are the same cutting sequence up to translation, and under this translation $b_{0}$ in $T^{\prime}$ gets sent to $b_{0}$ in $T^{\prime \prime}$.

Case 2: $\beta \alpha^{-1} \geq\left[\alpha^{-1}\right]$. Then $\beta_{2}=\left\{\alpha^{-1}\right\}-\left\{\beta \alpha^{-1}\right\}=\left\{\alpha^{-1}\right\}+\left[\alpha^{-1}\right]-\beta \alpha^{-1}$. So we may write $\mathrm{L}^{\prime \prime}$ as $y=\left\{\alpha^{-1}\right\}(x+1)-\beta \alpha^{-1}+\left[\alpha^{-1}\right]$. Thus $\mathrm{L}^{\prime \prime}$ is $\mathrm{L}^{\prime}$ raised by $\left[\alpha^{-1}\right]$ units and shifted one unit to the left. So $T^{\prime}$ and $T^{\prime \prime}$ are same cutting sequences up to translation, and $a_{-\left[\alpha^{-1}\right]}$ in $T^{\prime}$ is sent to $a_{0}$ in $T^{\prime \prime}$.

To prove the last assertion, note that

$$
\begin{aligned}
\mathrm{T} \text { is singular } & \Longleftrightarrow X \text { is tail equivalent to one of }\left\{-\alpha, 0^{+}, 0^{-}\right\} \\
& \Longleftrightarrow \beta \in \mathbb{Z}+\alpha \mathbb{Z} \\
& \Longleftrightarrow \mathrm{L} \text { passes through a point of } \mathbb{Z}^{2} .
\end{aligned}
$$

Lemma 3.3. When $x_{i}<a_{i}$ we have $T_{i}=S_{i}$, and when $x_{i}=a_{i}$ we have $T_{i}=S_{i-1}$. Proof. We will prove this by induction on $i$. When $i=1, T_{1}$ is either $\underbrace{\mathrm{b} \cdots \mathrm{b}}_{a_{1}}=S_{1}$ (if $x_{i}<a_{i}$ ), or $\mathrm{b}=S_{0}$ (if $x_{i}=a_{i}$ ). Suppose the result is true for $k-1$.

Case (i): $x_{k}<a_{k}$. Then

$$
T_{k}= \begin{cases}S_{k-2} \underbrace{S_{k-1} \cdots S_{k-1}}_{x_{k}} T_{k-1} S_{k-1} \cdots S_{k-1} & \text { if } x_{k-1}<a_{k-1} \\ T_{k-2} \underbrace{S_{k-1} \cdots S_{k-1}}_{a_{k}} & \text { if } x_{k-1}=a_{k-1} .\end{cases}
$$

In the first case $T_{k-1}=S_{k-1}$, so $T_{k}=S_{k}$; and in the second case $T_{k-2}=S_{k-2}$, since $x_{k-2}$ must be different from $a_{k-2}$. Thus $T_{k}=S_{k}$.

Case (ii): $x_{k}=a_{k}$. In this case $T_{k}=T_{k-1}=S_{k-1}$, since $x_{k-1} \neq a_{k-1}$.

Theorem 3.4 (Theorem $\mathrm{C}$ ). Let $T$ be a cutting sequence, $t$ a tile in $T$, and $x$ the coding sequence of $(T, t)$. Then the Smith sequence $\left\{T_{i}\right\}_{i=1}^{\infty}$ of $x$ equals the inflation sequence $\left\{W_{i}\right\}_{i=1}^{\infty}$ of $(T, t)$, i.e. $W_{i}=T_{i}$ for $i=1,2,3, \ldots$ 
Proof. Given a cutting sequence $\mathrm{T}$, let $\mathrm{T}_{1}=\mathrm{T}, \mathrm{T}_{2}, \mathrm{~T}_{3}, \ldots$ be the cutting sequences obtained from $\mathrm{T}_{1}$ by composition. We shall show that $\mathrm{T}_{k}$ can be obtained by rewriting $\mathrm{T}_{1}$ using the words $S_{k-2}$ and $S_{k-1}$.

We begin by replacing all a's in $\mathrm{T}_{1}$ by $S_{-1}$ and all b's in $\mathrm{T}_{1}$ by $S_{0}$. We have now rewritten $\mathrm{T}_{1}$ in the words $S_{-1}$ and $S_{0}$ (see 1.4).

Now replace all words $S_{-1} \underbrace{S_{0} \cdots S_{0}}_{a_{1}}$ with $S_{1}$. We now have a sequence of $S_{1}$ 's and $S_{0}$ 's. By making the substitution $S_{1} \mapsto$ b and $S_{0} \mapsto$ a we have the cutting sequence $\mathrm{T}_{2}$.

If we now take our sequence of $S_{1}$ 's and $S_{0}$ 's and replace each word $S_{0} \underbrace{S_{1} \cdots S_{1}}_{a_{2}}$ by $S_{2}$, we have a sequence of $S_{2}$ 's and $S_{1}$ 's. The map that takes $S_{2} \mapsto$ b and $S_{1} \mapsto$ a transforms $\mathrm{T}$ into $\mathrm{T}_{3}$.

Suppose we have performed this substitution $k-1$ times and have rewritten T as a sequence of $S_{k-1}$ 's and $S_{k-2}$ 's which becomes $T_{k}$ under the map $S_{k-1} \mapsto$ b and $S_{k-2} \mapsto$ a. Let us show that if we make one more iteration we obtain a rewriting of T as a sequence of $S_{k-1}$ 's and $S_{k}$ 's which becomes $\mathrm{T}_{k+1}$ under the map $S_{k} \mapsto$ b and $S_{k-1} \mapsto$ a.

First notice that because of the bijection with $\mathrm{T}_{k}$ there are in $\mathrm{T}$ either $a_{k}$ or $1+a_{k} S_{k-1}$ 's between adjacent $S_{k-2}$ 's. Thus the rewrite rule $S_{k-2} \underbrace{S_{k-1} \cdots S_{k-1}}_{a_{k}}$ $\mapsto S_{k}$ can be performed. Moreover, by transforming this rewrite rule by $S_{k-2} \mapsto$ a and $S_{k-1} \mapsto$ b we get exactly the composition rule for passing from $\mathrm{T}_{k}$ to $\mathrm{T}_{k+1}$; i.e. the following diagrams commute:
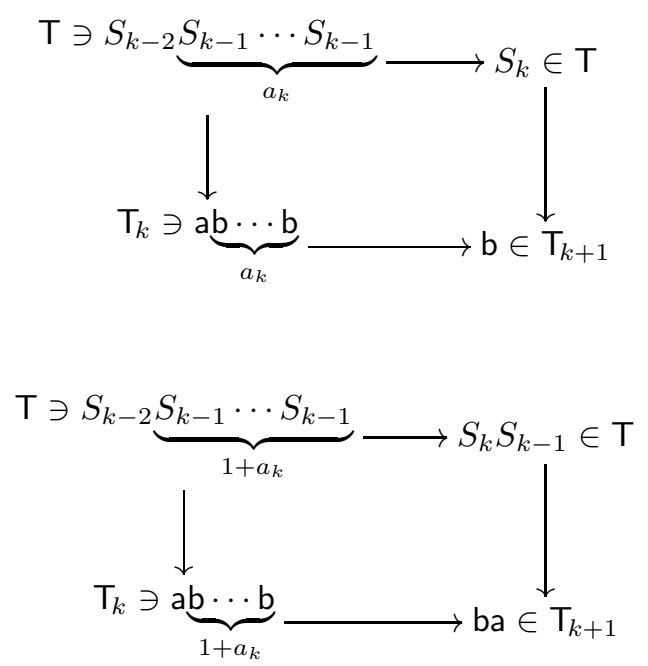

where in the left hand columns we are using the rule $S_{k-2} \mapsto$ a and $S_{k-1} \mapsto$ b, and in the right hand columns we are using the rule $S_{k-1} \mapsto$ a and $S_{k} \mapsto$ b. This establishes our claim.

We shall prove that $W_{i}=T_{i}$ by induction on $i$. For $i=1$ we have either $W_{1}=\underbrace{\mathrm{a} \cdots \mathrm{b}}_{a_{1}}=T_{1}$ when $x_{1}<a_{1}$, or $W_{1}=\mathrm{b}=T_{1}$ when $x_{1}=a_{1}$. So let us suppose that $W_{i}=T_{i}$ for $1 \leq i \leq k$, and show that $W_{k+1}=T_{k+1}$. We have three cases to 
consider:

(i) $x_{k+1}=a_{k+1}$.

(ii) $x_{k+1}<a_{k+1}$ and $x_{k}<a_{k}$.

(iii) $x_{k}=a_{k}$.

Case (i): In this case $\mathrm{t}_{k+1}=\mathrm{b}$ and appears in $\mathrm{T}_{k+1}$ as $\cdots \mathrm{a} \underbrace{\mathrm{b} \cdots \mathrm{b}}_{a_{k+1}} \mathrm{~b} \cdots$

This $\mathrm{b}$ gets sent to an a in $\mathrm{T}_{k+2}$, so no additional letters are picked up; i.e. $W_{k+1}=W_{k}=T_{k}=T_{k+1}$.

Case (ii): In this case $x_{k+1}<a_{k+1}$ and $x_{k}<a_{k}$, so $\mathrm{t}_{k+1}=\mathrm{b}$ in $\cdots \mathrm{a} \underbrace{\mathrm{b} \cdots \mathrm{b}}_{x_{k+1}}$

$\mathrm{b} \mathrm{b} \cdots \mathrm{b}$, which gets mapped to $\mathrm{b}$ in $\mathrm{T}_{k+2} . W_{k+1}$ is the inverse image in $\mathrm{T}_{1}$ of this b. Under the rewrite rule $S_{k} \mapsto \mathrm{b}, S_{k-1} \mapsto \mathrm{a}: \mathrm{T}_{1} \rightarrow \mathrm{T}_{k+1}$, the corresponding interval in $\mathrm{T}_{1}$ is $\cdots S_{k-1} \underbrace{S_{k} \cdots S_{k}}_{x_{k+1}} T_{a_{k+1}-x_{k+1}-1}^{S_{k} \cdots S_{k}} \cdots$ (where we know that $T_{k}=S_{k}$ because $x_{k}<a_{k}$ ). Thus

$$
W_{k+1}=S_{k-1} \underbrace{S_{k} \cdots S_{k}}_{x_{k+1}} T_{a_{k+1}-x_{k+1}-1}^{S_{k} \cdots S_{k}}=T_{k+1}
$$

Case (iii): In this case $x_{k}=a_{k}$ and $x_{k+1}=0$. Thus $\mathrm{t}_{k+1}=\mathrm{a}$ in $\cdots \mathrm{a} \underbrace{\mathrm{b} \cdots \mathrm{b}}_{a_{k+1}}$ $\cdots \subseteq \mathrm{T}_{k+1}$. This segment of letters gets mapped to a b in $\mathrm{T}_{k+2}$, so $W_{k+1}$ is the inverse image in $\mathrm{T}_{1}$ of $\underbrace{\mathrm{b} \cdots \mathrm{b}}_{a_{k+1}}$ in $\mathrm{T}_{k+1}$. Using the rewrite rule $S_{k-1} \mapsto$ a and $S_{k} \mapsto \mathrm{b}$, the corresponding interval in $\mathrm{T}_{1}$ is

$$
W_{k+1}=S_{k-1} \underbrace{S_{k} \cdots S_{k}}_{a_{k+1}}=T_{k-1} \underbrace{S_{k} \cdots S_{k}}_{a_{k+1}}=T_{k+1}
$$

since $x_{k-1}<a_{k-1}\left(\right.$ as $\left.x_{k}=a_{k}\right)$.

Theorem 3.5 (Theorem D). Let $\sim$ be the equivalence relation on $X_{\alpha}$ generated by tail equivalence, and let $-\alpha \sim 0^{+} \sim 0^{-}$. Let $\left(T_{1}, t_{1}\right)$ and $\left(T_{2}, t_{2}\right)$ be in $\mathcal{T}_{\alpha}^{\bullet}$, and $x_{1}$ and $x_{2}$ in $X_{\alpha}$ the corresponding coding sequences. Then $x_{1} \sim x_{2}$ if and only if $T_{1} \approx T_{2}$.

Proof. Suppose $(\mathrm{T}, \mathrm{t})$ and $\left(\mathrm{T}^{\prime}, \mathrm{t}^{\prime}\right)$ are in $\mathcal{T}_{\alpha}^{\bullet}$ with corresponding coding sequences $\left(x_{i}\right)$ and $\left(x_{i}^{\prime}\right)$ in $X_{\alpha}$. Suppose that $(\mathrm{T}, \mathrm{t})$ and $\left(\mathrm{T}^{\prime}, \mathrm{t}^{\prime}\right)$ are equivalent. Then either they are both singular, in which case $\left(x_{i}\right)$ and $\left(x_{i}^{\prime}\right)$ are in the tail equivalence class of $\left\{-\alpha, 0^{+}, 0^{-}\right\}$by Theorem $\mathrm{B}$, or neither is singular, in which case $\left(x_{i}\right)$ and $\left(x_{i}^{\prime}\right)$ are tail equivalent, again by Theorem B.

Suppose that $\left(x_{i}\right)$ and $\left(x_{i}^{\prime}\right)$ are equivalent. Then there are two cases.

Case (i): $\left(x_{i}\right)$ and $\left(x_{i}^{\prime}\right)$ are tail equivalent. We shall show that that there is $\tilde{\mathrm{t}}^{\prime}$ in $\mathrm{T}^{\prime}$ such that $(\mathrm{T}, \mathrm{t})=\left(\mathrm{T}^{\prime}, \tilde{\mathrm{t}}^{\prime}\right)$. Suppose that $x_{i}=x_{i}^{\prime}$ for $i \geq k$. Let $\mathrm{T}_{i}$ for $i=1,2,3, \ldots$ and $\mathbf{T}_{i}^{\prime}$ for $i=1,2,3, \ldots$ be the sequences obtained from $\mathbf{T}$ and $\mathbf{T}^{\prime}$ respectively by composition. Also let $\mathrm{t}_{k} \in \mathrm{T}_{k}$ and $\mathrm{t}_{k}^{\prime} \in \mathrm{T}_{k}^{\prime}$ be the letters which after $k-1$ compositions absorb $\mathrm{t}$ and $\mathrm{t}^{\prime}$ respectively. The coding sequences of $\left(\mathrm{T}_{k}, \mathrm{t}_{k}\right)$ and $\left(\mathrm{T}_{k}^{\prime}, \mathrm{t}_{k}^{\prime}\right)$ are respectively $\left(x_{k}, x_{k+1}, x_{k+2}, \ldots\right)$ and $\left(x_{k}^{\prime}, x_{k+1}^{\prime}, x_{k+2}^{\prime}, \ldots\right)$, and hence are equal. Thus by Theorem $\mathrm{C}$ the inflation sequence of $\mathrm{t}_{k}$ in $\mathrm{T}_{k}$ is equal to the inflation sequence of $\mathrm{t}_{k}^{\prime}$ in $\mathrm{T}_{k}^{\prime}$. Since these cutting sequences are non-singular, they are the 
union of the corresponding inflation sequences. Hence $\left(\mathrm{T}_{k}, \mathrm{t}_{k}\right)=\left(\mathrm{T}_{k}^{\prime}, \mathrm{t}_{k}^{\prime}\right)$. Now by the argument presented at the beginning of the proof of Theorem $\mathrm{C}$ let us rewrite T as a sequence of $S_{k-1}$ 's and $S_{k-2}$ 's so that, under the transformation $S_{k-1} \mapsto$ b and $S_{k-2} \mapsto \mathrm{a}, \mathrm{T}$ is transformed into $\mathrm{T}_{k}$. Let us do the same for $\mathrm{T}^{\prime}$ and $\mathrm{T}_{k}^{\prime}$. Since $\left(\mathrm{T}_{k}, \mathrm{t}_{k}\right)=\left(\mathrm{T}_{k}^{\prime}, \mathrm{t}_{k}^{\prime}\right)$, we see that, when written as a sequence of $S_{k-1}$ 's and $S_{k-2}$ 's, T and $\mathrm{T}^{\prime}$ are equal, and that $\mathrm{t}$ and $\mathrm{t}^{\prime}$ lie in the same word (either a $S_{k-1}$ or a $S_{k-2}$ ). Hence $(\mathrm{T}, \mathrm{t})=\left(\mathrm{T}^{\prime}, \tilde{\mathrm{t}}^{\prime}\right)$, for some letter $\tilde{\mathrm{t}}^{\prime}$ in $\mathrm{T}^{\prime}$ no more distant from $\mathrm{t}$ than $\left|S_{k-1}\right|$.

Case (ii) : $\left(x_{i}\right)$ and $\left(x_{i}^{\prime}\right)$ are in the tail equivalence class of $\left\{-\alpha, 0^{+}, 0^{-}\right\}$. By Theorem $\mathrm{B}$ we know that $\mathrm{T}$ and $\mathrm{T}^{\prime}$ come from lines which pass through a point of $\mathbb{Z}^{2}$, and thus both $\mathrm{T}$ and $\mathrm{T}^{\prime}$ are singular. By Theorem $\mathrm{C}$, we know that up to a translation there are only the two singular cutting sequences $\mathrm{T}^{+}$and $\mathrm{T}^{-}$discussed in 1.6, and these are conjugate. Hence $T \approx T^{\prime}$.

\section{REFERENCES}

[AP] J. E. Anderson and I. F. Putnam, Topological Invariants for Substitution Tilings and their Associated C*-algebras, Ergodic Thry. and Dynamical Systems, 18 (1989) 509-537. MR 2000a:46112

[JB] Jean Bernoulli (III), (1744-1807), Recueil pour les Astromomes, tome 1, Berlin, chez l'auteur, (1771).

[тсв] T. C. Brown, Descriptions of the Characteristic Sequence of an Irrational Number, Canad. Math. Bull. 36 (1993) 15-21. MR 94g:11051

[NDB 1 ] N. G. de Bruijn, Sequences of zeros and ones generated by special production rules, Indag. Math. 43 (1981), 27-37. MR 82i:10074

[NDB 2$]$ N. G. de Bruijn, Updown generation of Beatty sequences, Indag. Math. 51 (1989), 385-407. MR 91d:11006

[EBC] E. B. Christoffel, Observatio Arithmetica, Annali di Mathematica, (2) 6 (1875) 148-152.

[AC] A. Connes, Noncommutative Geometry, San Diego, Academic Press, 1994. MR 95j:46063

[GS] B. Grünbaum and G. C. Shephard, Tilings and Patterns, New York, W. H. Freeman and Company, 1997. MR 90a:52027

[LP] W. F. Lunnon and P. A. B. Pleasants, Characterization of two-distance sequences, J. Austral. Math. Soc., Ser. A, 53 (1992), 198-218. MR 93h:11027

[JAM] J. A. Mingo, C*-algebras associated with one dimensional almost periodic tilings, Comm. Math. Phys. 183 (1997), 307-337. MR 98j:46058

[MH] M. Morse and G. Hedlund, Symbol Dynamics II, Sturmian Sequences, Amer. J. Math. 62 (1940), 1-42. MR 1:123d

[wP] W. Parry, On the $\beta$-expansions of real numbers, Acta. Math. Acad. Sci. Hung., 11, (1960), 401-416. MR 26:288

[AR] A. Rényi, Representations for real numbers and their ergodic properties, Acta. Math. Acad. Sci. Hung., 8 (1957), 477-493. MR 20:3843

[EAR] E. Arthur Robinson, Jr, The Dynamical Properties of Penrose Tilings, Trans. Amer. Math. Soc., 348 (1996), 4447-4464. MR 97a:52041

[Ms] M. Senechal, Quasicrystals and Geometry, Cambridge, Cambridge University Press, 1995. MR 96c:52038

[CS] C. Series, The Geometry of Markoff Numbers, Math. Intelligencer, 7 (1985) 20-29. MR 86j:11069

[HJss] H. J. S. Smith, A Note on Continued Fractions, Messenger of Math., (2) 6 (1876) 1-14.

Department of Mathematics and Statistics, Queen's University, Kingston, Ontario K7L 3N6, CANADA

E-mail address: mingoj@mast.queensu.ca 